\title{
Knockdown of PLCB2 expression reduces melanoma cell viability and promotes melanoma cell apoptosis by altering Ras/Raf/MAPK signals
}

\author{
HUAHUI ZHANG ${ }^{1}$, TAO XIE $^{2}$, YONGJIE SHUI ${ }^{3}$ and YIYING QI ${ }^{4}$ \\ ${ }^{1}$ Department of Plastic Surgery, Zhejiang Hospital, Hangzhou, Zhejiang 310007; \\ ${ }^{2}$ Department of Orthopedics, Affiliated Hangzhou First People's Hospital, Zhejiang University School of Medicine, \\ Hangzhou, Zhejiang 310006; Departments of ${ }^{3}$ Radiotherapy and ${ }^{4}$ Orthopedics, The Second Affiliated Hospital, \\ Zhejiang University School of Medicine, Hangzhou, Zhejiang 310009, P.R. China
}

Received February 26, 2019; Accepted May 29, 2019

DOI: $10.3892 / \mathrm{mmr} .2019 .10798$

\begin{abstract}
Malignant melanoma has the highest malignancy rate among all skin cancer and is characterized by an insidious onset, high invasion and poor patient prognosis. Yet, the mechanisms involved remain unclear and warrant further investigation. Based on bioinformatic analysis, phospholipase $\mathrm{C} \beta 2$ (PLCB2) has been found to be correlated with melanoma growth. The present study was the first to demonstrate that $P L C B 2$ is a key factor affecting melanoma proliferation and apoptosis. Here, microarray datasets from the publicly available Gene Expression Omnibus (GEO) database were employed, and gene set enrichment analysis (GSEA) was introduced to identify candidate transcription factors. PLCB2 was identified as a crucial gene in the protein-protein interaction (PPI) network. The expression of PLCB2 mRNA in various cancer lines was analyzed by reverse transcription-polymerase chain reaction (RT-PCR). In addition, the proliferation ability and apoptosis rate in human melanoma cells overexpressing or not overexpressing $P L C B 2$ were assessed using colony formation assay, flow cytometry and the Cell Counting Kit-8 (CCK-8) assay. Cell viability and apoptosis-related factors, such as p53, Bcl-2, Bax and caspase-3 were significantly regulated. Knockdown of PLCB2 suppressed the activation of the Ras/Raf/MAPK signaling pathway. In conclusion, knockdown of PLCB2 suppressed cell viability and promoted cell apoptosis by activating the Ras/Raf/MAPK pathway. Thus, PLCB2 may utilized as a potential therapeutic target in patients with melanoma.
\end{abstract}

Correspondence to: Dr Huahui Zhang, Department of Plastic Surgery, Zhejiang Hospital, 12 Lingyin Road, Hangzhou, Zhejiang 310007, P.R. China

E-mail: syjhz@zju.edu.cn

Key words: melanoma, phospholipase $\mathrm{C} \beta 2$, proliferation, apoptosis, Ras/Raf/MAPK

\section{Introduction}

Melanoma, also known as malignant melanoma, is the most dangerous type of skin cancer, with a high degree of malignancy and poor patient prognosis. It is commonly observed on the skin and mucosa, originating mainly from epithelial melanocytes $(1,2)$. Although melanoma accounts for approximately $10 \%$ of all skin cancers, it accounts for approximately $80 \%$ of the all skin cancer-related deaths (1). The etiopathogenesis of melanoma is complex, and most common etiologies involve an endocrine abnormality, genetic variation or ultraviolet irradiation exposure (3). The extent of malignant melanoma has a positive relationship with the number of tumor metastatic sites. Studies have shown that the main reason for the death of patients with malignant melanoma is tumor metastases to the liver, lung, bone and brain (4-7). The authors conclude there are many means of melanoma metastasis, e.g., lymphatic metastasis, blood metastasis and implantation metastasis. Melanoma is characterized by malignant proliferation, immune escape, metastasis and angiogenesis (8). Accordingly, the options for melanoma treatment are limited and are ineffective to improve the quality of life or survival of patients.

Targeted therapy for mutated genes, abnormal genes and their signal transduction systems has been applied to clinical therapy gradually, and it may benefit patients with melanoma; however, there are still many issues associated with melanoma treatment, e.g, drug resistance and drug insensitivity (9). Taken together, there exists an urgent need to perform in-depth research on the pathogenesis of melanoma to further explore new targets of drug action to be used in the clinic.

According to a previous report, $P L C B 2$ is a critical regulator of platelet responses upon activation and takes part in biological behaviors, e.g., tumorigenesis, cell growth and activation of neutrophils by pro-inflammatory mediators $(10,11)$. In addition, bioinformatic analysis has revealed that $P L C B 2$ is highly expressed in melanoma cells with high $A B C B 5$ expression. Further reports have shown that $P L C B 2$ is significantly expressed in kidney renal clear cell carcinoma and bladder cancer $(12,13)$. Thus, PLCB2 may be a novel gene involved in the incidence and development of melanoma. 
The Ras/Raf/MAPK signaling pathway is closely associated with cell proliferation and differentiation and takes part in the development and progression of skin malignant tumors $(14,15)$. Ras, as an oncogene, plays a pivotal role in cell life activities and mediates tumorigenesis by the Raf/MEK/ERK and PI3K/Akt signaling pathways (16). Raf leads to the cascade of the Ras/Raf/MEK1/2/-ERK1/2 signaling pathway. ERK is a $M A P K$ kinase and plays an important role in cellular signal transduction in the Ras/Raf/MAPK signaling pathway (17). Combined with our previous studies, our results suggest that the $M A P K$ signaling pathway is closely associated with melanoma progression. Therefore, the present study was conducted to ascertain whether PLCB2 influences cell viability and apoptosis by activation of the Ras/Raf/MAPK signaling pathway.

\section{Materials and methods}

Bioinformatic analysis. To assess the expression of PLCB2 in human melanoma cells, the online microarray profiling data set, GEO accession no. GSE38290 (18), which includes 12 samples, was used. Each of the following 4 groups had three replicates: A375 pSUPER-retro-puro-Vector vs. A375 pSUPER-retro-puro-ABCB5-KD; and G3361 pSUPER-retro-puro-shCNTRL vs. G3361 pSUPER-retro-puro-ABCB5-KD. To renormalize the Affymetrix array data, the authors averaged the intensity values of 3 probes per sample in each data set as these probes adopted the same internal control. For each data set, the average expression level of PLCB2 in the A375 pSUPER-retro-puro-Vector and G3361 pSUPER-retro-puro-shCNTRL groups was set to 1 , and the expression of PLCB2 in the A375 pSUPER-retro-puro-ABCB5-KD and G3361 pSUPER-retro-puro-ABCB5-KD groups was adjusted accordingly. Using the 'gplots' package of R (https://cran.r-project. org/web/packages/gplots/), heatmap visualization of data matrices was performed. Using the variance stabilizing transformation methods, the authors conducted principal component analysis of RNA-Seq results in the 'wilcoxTest' package of Bioconductor (http://bioconductor.org/), and then plotted the first two principal components. Volcano plots were derived from 'wilcoxTest'-based differential gene expression analysis.

Gene set enrichment analysis (GSEA). The Broad Institute website (http://www.broadinstitute.org/gsea/index.jsp) was used to conduct the gene set enrichment analysis (19). This analysis covers versions compatible with Java, $\mathrm{R}$ or Gene Pattern. The authors conducted all GSEAs presented by implanting the Java GSEA. No experiments (covering human material, humans, or animals) were conducted. Accordingly, no ethical approval was required.

Protein-protein interaction (PPI) network analysis. The STRING database (www.string-db.org) (20) was used to collect and integrate known and predicted protein-protein association data for 13 genes. In the present study, STRING was used to construct the PPI network of 13 genes with a minimum required interaction score of 0.7 . In the present study, the crucial genes were identified based on four different centrality measures, e.g. betweenness centrality (BC), degree centrality (DC), eigenvector centrality (EGC), and closeness centrality (CC).
According to the centrality values of genes in the PPI network, the top 3 genes were identified as crucial genes.

Cell lines and cell culture. The normal human epidermal melanocyte (NHEM) cell line was provided by Cell Applications Inc. and cultured in M2 medium. The China Center for Type Culture Collection (Wuhan, China) provided human melanoma cell lines (451Lu, Mel Ju, Mel 928, SK-MEL-28, SK-MEL-2, Hs294T, A375 and WM35) which were cultivated in Dulbecco's modified Eagle's medium (DMEM) (Gibco; Thermo Fisher Scientific, Inc.) supplemented with $10 \%$ fetal bovine serum. All cell lines were incubated at $37^{\circ} \mathrm{C}$ with $5 \% \mathrm{CO}_{2}$ in a humidified environment.

RNA interference. The authors adopted negative control small interfering RNA (siRNA) (5'-UUCUCCGAACGUGUCACG UTT-3) and an siRNA against PLCB2 (5'-CCCTGCCCA GCCCTCAGGGGATCAGT-3') (GenePharma). The siRNAs were transfected into A375 cells using Lipofectamine ${ }^{\mathrm{TM}} 2000$ transfection reagent (Invitrogen; Thermo Fisher Scientific, Inc.) (21). After transfection, the cells underwent $48 \mathrm{~h}$ of culture before treatment according to the manufacturer's instructions.

Transfection of cells with the PLCB2-overexpressing plasmid vector. A PLCB2 overexpression vector (pcDNA3.1/PLCB2-HisB) was used for the transfection studies. Using Lipofectamine ${ }^{\mathrm{TM}} 2000$ transfection reagent (Invitrogen; Thermo Fisher Scientific, Inc.) following the manufacturer's instructions (22), all transfection experiments were performed. Cells $\left(1 \times 10^{3}\right)$ were plated in 24-well plates and allowed to grow until reaching $80-90 \%$ confluency. The plasmid DNA and Lipofectamine $(4 \mu \mathrm{l} / \mathrm{ml}$ of transfection medium) were diluted in Opti-MEM reduced serum medium (Thermo Fisher Scientific, Inc.) separately and incubated for $10 \mathrm{~min}$ at room temperature. The diluted solutions were then mixed and incubated for $30 \mathrm{~min}$. Subsequently, $4 \mu \mathrm{g}$ plasmid and $10 \mu \mathrm{l}$ reagent were added to each well containing cells, mixed gently, and the cells were then stored in a $\mathrm{CO}_{2}$ incubator for $48 \mathrm{~h}$ at $37^{\circ} \mathrm{C}$.

Cell viability assays. Cell viability was assessed using the Cell Counting Kit-8 assay (Sigma-Aldrich; KGaA), following the manufacturer's instructions. Briefly, cells were seeded in 96-well plates at a density of $4 \times 10^{3}$ cells per well in $100 \mu 1$ of growth medium, and allowed to attach overnight. After achieving attachment for $24 \mathrm{~h}$, the cells were treated with oxaliplatin $(\mu \mathrm{M})$ at different concentrations for different periods. At the end of the treatment, $10 \mu \mathrm{l}$ of tetrazolium substrate was added to each well of the plate. The plates were then stored at $37^{\circ} \mathrm{C}$ for $1 \mathrm{~h}$ and the optical density (OD) was measured at $450 \mathrm{~nm}$ with a Bio-Rad ELISA microplate reader (Bio-Rad 680; Bio-Rad Laboratories, Inc.).

Colony formation assay. For the colony formation assay, cells were seeded at 300 cells/well on 6-well plates in triplicate, and maintained in complete culture medium for 12 days until obvious colonies formed. Colonies were then fixed with $70 \%$ ethanol for $5 \mathrm{~min}$ at room temperature and stained with Coomassie blue dye for $5 \mathrm{~min}$ as well. A colony containing more than 50 cells was considered a colony and was counted. 
Colony number was determined under an Olympus fluorescence microscope equipped with MicroPublisher 3.3 RTV CCD camera.

Analysis of apoptosis by flow cytometry. With the use of Annexin V-FITC apoptosis detection reagent (BD Biosciences) following the manufacturer's instructions, cell apoptosis was detected. Briefly, cells were washed twice with cell staining buffer and resuspended in binding buffer at a density of $1 \times 10^{5}$ cells $/ 100 \mu l$. To $100 \mu \mathrm{l}$ of the cell suspension, $5 \mu \mathrm{l}$ of Annexin V-FITC was added, and the cells were incubated at ambient temperature for $15 \mathrm{~min}$, followed by addition of another $400 \mu$ of binding buffer. Subsequently, the cell apoptosis was detected with the use of flow cytometry (LSR II, BD Biosciences), and the data were analyzed using FlowJo software (FlowJo LLC, USA).

RNA extraction and quantitative real-time PCR. Total RNA was extracted from A375 cells overexpressing or not overexpressing PLCB2 using TRIzol reagent (Invitrogen; Thermo Fisher Scientific, Inc.) following the manufacturer's instructions. The RNA concentration was ascertained using UV spectrophotometry. Total RNA $(0.5 \mu \mathrm{g})$ was employed to yield cDNA with SuperScript II reverse transcriptase (Invitrogen; Thermo Fisher Scientific, Inc.) following the specifications of the manufacturer $(23,24)$; overall RNA $(0.5 \mu \mathrm{g})$ was employed. Genes of interest were amplified by real-time PCR using a LightCycler TaqMan Master kit (Roche Diagnostics) following the specifications of the manufacturer and primers designed by Roche Diagnostics. The following primers were employed: $p 53$ forward, 5'- CTGGCATTTGCACCTACCTC-3' and reverse, 5'-TAACCAGCTGCCCACTGTA-3' (product 176 bp); Bax forward, 5'-TTTGCTTCAGGGTTTCATCC-3' and reverse, 5'-AGACCTGCCGTTGAAGTTGAC-3' (product $120 \mathrm{bp}$ ); $\mathrm{Bcl}$-2 forward, 5'-CGCCAACATTCTCTCCACAG-3' and reverse, 5'-CTGGGCCAGAGCTACATCTT-3' (product $119 \mathrm{bp}$ ); and GAPDH forward, 5'-ACCCAGAAGACTGTGGATGG-3' and reverse, 5'-TCAGCTCAGGGATGACCTTG-3' (product $124 \mathrm{bp}$ ). The final reaction volume reached $10 \mu \mathrm{l}$, and for PCR (Roche Diagnostics), a LightCycler instrument was utilized. The following PCR conditions were used: $15 \mathrm{~min}$ at $95^{\circ} \mathrm{C}$, followed by 40 cycles for $10 \mathrm{sec}$ at $95^{\circ} \mathrm{C}, 30 \mathrm{sec}$ at $60^{\circ} \mathrm{C}$, and $1 \mathrm{sec}$ at $72^{\circ} \mathrm{C}$, and 1 cycle of cooling for $30 \mathrm{sec}$ at $50^{\circ} \mathrm{C}$. The authors normalized the samples to the GAPDH gene. The authors obtained the corresponding expression using the $2^{-\Delta \Delta C q}$ formula (25).

Protein extraction and western blotting. At $48 \mathrm{~h}$ post transfection, the cells were lysed using NP-40 lysis buffer (50 mM Tris, $\mathrm{pH} 7.5,150 \mathrm{mM} \mathrm{NaCl}, 1.0 \% \mathrm{NP}-40,2 \mathrm{mM}$ EDTA, $10 \mathrm{mM} \mathrm{NaF}$, $1 \mathrm{mM} \mathrm{Na}_{3} \mathrm{VO}_{4}$ and $2 \mathrm{mM}$ PMSF). As previously described (26), the concentration of protein samples underwent measurement with the use of a BCA kit (Solarbio) in line with the instructions of the manufacturer. An amount of $50 \mu \mathrm{l}$ of cell lysates was loaded on $8 \%$ SDS-PAGE gel and then transferred onto a polyvinylidene difluoride (PVDF) membrane (Millipore). After being blocked using 5\% non-fat dry milk powder in PBS with $0.1 \%$ Tween- 20 for $1 \mathrm{~h}$, the membrane underwent incubation using the indicated antibodies at $4^{\circ} \mathrm{C}$ overnight. After being washed using Tris-buffered saline-Tween-20 (TBST) 3 times, the membranes underwent incubation using horseradish peroxidase (HRP)-conjugated goat anti-rabbit (cat. no. 6721, dilution of 1:5,000; Abcam) or goat anti-mouse (cat. no. 6789, dilution of 1:2,000; Abcam) secondary antibodies at ambient temperature for $1 \mathrm{~h}$ and washed with TBST 3 times. Finally, the proteins underwent visualization with an ECL Western blotting detection kit (Amersham Biosciences) in line with the manufacturer's instructions. Quantification of the bands was performed via densitometry and the ImageJ Pro 6.0 analyses systems (National Institutes of Health, NIH, Bethesda, MD, USA). The following antibodies were employed: p53 (cat. no. 32389, dilution 1:500; Abcam), c-caspase-3 (cat. no. 49822, dilution 1:800; Abcam), Bax (cat. no. 77566, dilution 1:800; Abcam), Bcl-2 (cat. no. 32124, dilution 1:1,000; Abcam), Ras (cat. no. 52939, dilution 1:800; Abcam), Raf (cat. no. 200653, dilution 1:800; Abcam), p-ERK1/2 (CST cat. no. 4370, dilution 1:500; Cell Signaling Technology, Inc.), ERK1/2 (CST cat. no. 46955, dilution 1:1,000; Cell Signaling Technology, Inc.) and GAPDH (cat. no. 181602, dilution 1:2,000; Abcam).

Statistical analysis. Statistical analysis was conducted with the use of SPSS 18.0 software (SPSS, Inc., Chicago, IL, USA). Data are shown as the mean \pm standard deviation (SD). Statistical significance was ascertained by Dunnett's -test or Tukey's test. A P-value $<0.05$ was regarded as indicative of statistical significance.

\section{Results}

High expression of PLCB2 in human melanoma cell lines. The authors performed heatmap analysis of the expression profiles in ABCB5 highly expressing human melanoma cell lines (A375 and G3361 cells) in the GEO database (GSE38290). Amplified aRNA from each sample was subjected to microarray hybridization, and 14,209 extracted genes were analyzed by hierarchical clustering, which revealed distinct expression patterns in human melanoma cells with high $A B C B 5$ expression (experimental group) and human melanoma cells with empty vectors (control group) (Fig. 1A). A volcano plot of the identified quality-controlled genes $(\mathrm{P}<0.05$, fold change $>2)$ is presented in Fig. 1B. Our data identified 289 upregulated and 56 downregulated mRNAs in human melanoma cells with high $A B C B 5$ expression compared with matched human melanoma cells with empty vectors (Fig. 1B). GSEA showed that GNG13, PLCB2, EDNRA, LPAR4, PIK3R3, PRKCG, MMP2, PAX8, $P P A R G, F G F 7$ and $P T G E R 4$ were significantly enriched in Cell growth, MAPK cascade and Cancer-related signaling pathways (Fig. 1C-E). Thus, network analysis of the 13 genes identified in the present study was performed to predict functional gene-gene interactions with the use of the STRING database (www. string-db.org) (Fig. 1F). PLCB2 was identified as a crucial gene in the PPI network (Fig. 1G). In addition, PLCB2 was found to be significantly highly expressed in human melanoma cell lines, as determined by RT-PCR analysis (Fig. 1H).

PLCB2 affects cell viability and apoptosis. To further confirm the effect of $P L C B 2$ on the proliferation and apoptosis of human melanoma cells, A375 cells were transfected with overexpressing or not overexpressing PLCB2 plasmids. Efficiency of the PLCB2 siRNA and the overexpressing plasmid was examined by RT-PCR and western blot analysis (Fig. 2A). 
A
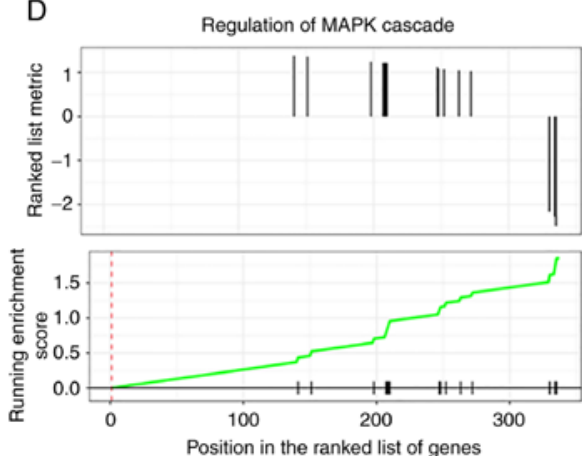

$\mathrm{F}$

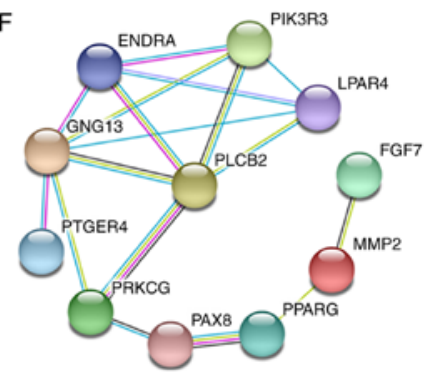

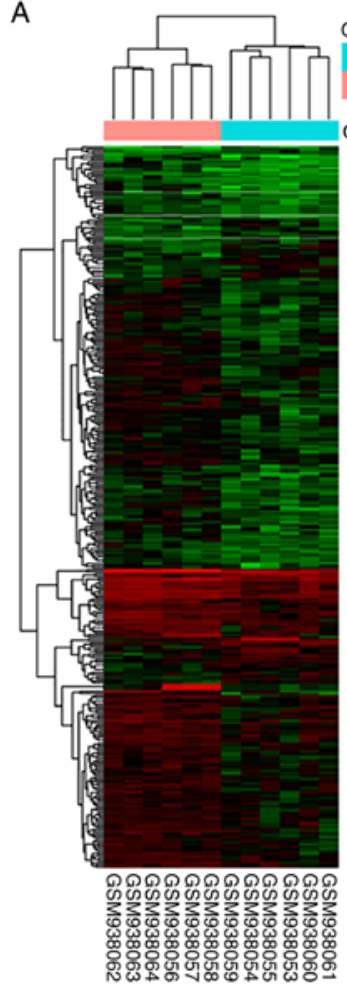

B
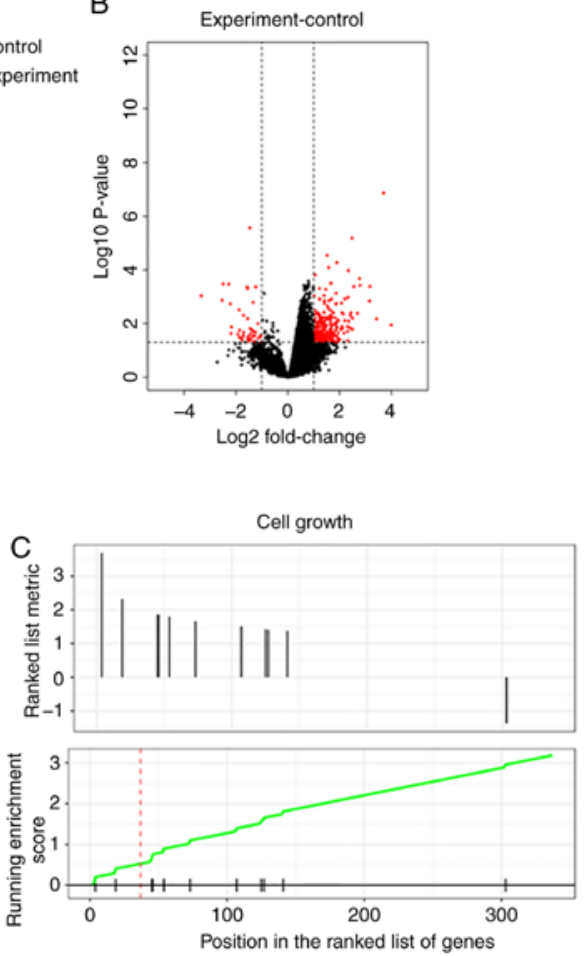

$\mathrm{E}$
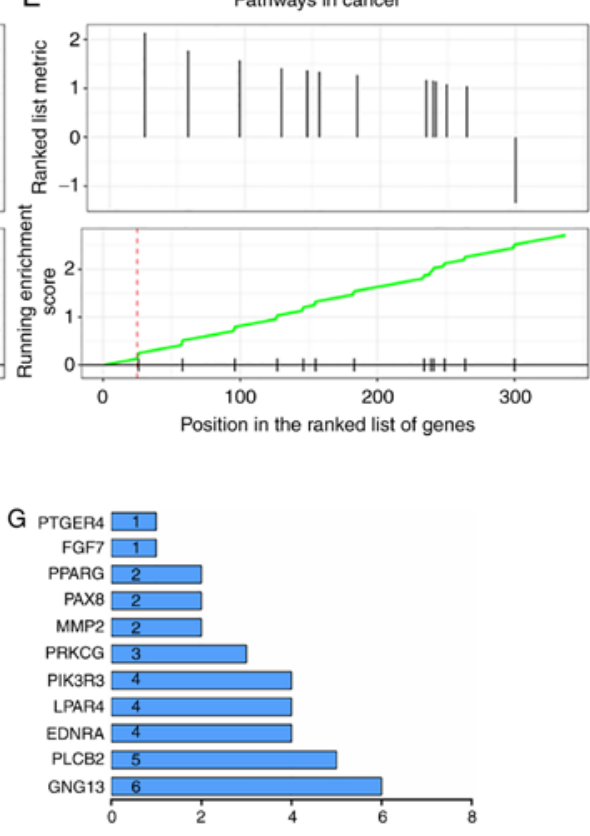

Rel. PLCB2 mRNA expression

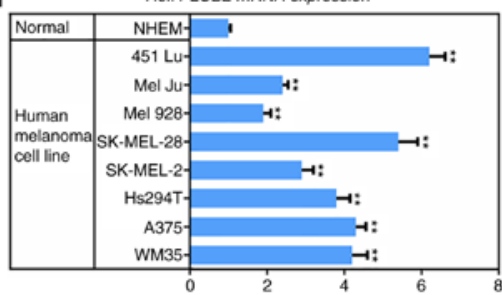

Figure 1. PLCB2 expression in malignant melanoma cell lines. (A) The heatmap analysis was performed by R software with wilcoxTest package (P<0.05 and log2 fold change $>1$ ) to illustrate miRNA expression profiles in the GEO database (GSE38290). (B) Volcano plot of DEGs. DEGs with log2 fold change $>1.5$ are shown in red. GSEA analysis was performed by Broad Institute website to show significantly enriched gene sets in melanoma mainly enriched in (C) cell growth, (D) regulation of MAPK cascade and (E) pathways in cancer. (F) The diagram of the PPI network. Co-expression network of 11 genes. (G) Core protein histogram. The vertical coordinates are the gene names, and the horizontal coordinates are the number of adjacent genes, and the height represents the number of line connected genes. $(\mathrm{H})$ mRNA expression level of PLCB2 in human melanoma cell lines was detected by RT-PCR. GAPDH was used as a load control. Data are presented as the mean \pm standard deviation. ${ }^{* *} \mathrm{P}<0.01$ vs. the NHEM cell group. PLCB2, phospholipase $\mathrm{C} \beta 2$; DEGs, differentially expressed genes; PPI-protein-protein interaction. 
A

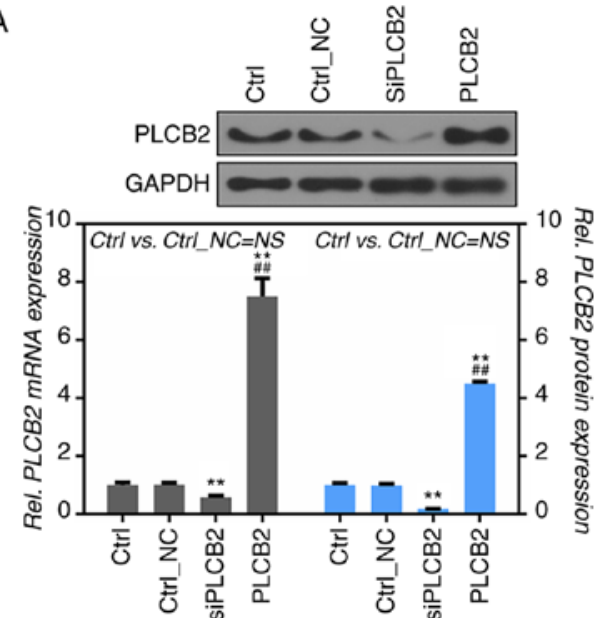

B

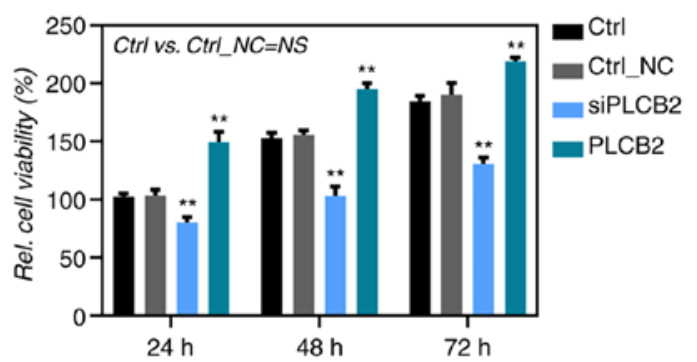

Figure 2. PLCB2 affects cell viability. (A) The transfection efficiency of the PLCB2 interference plasmid (siPLCB2) and PLCB2 overexpression plasmid (PLCB2) was confirmed by RT-PCR and western blot assay in A375 cells. (B) CCK-8 assay was used to detect cell viability at 24, 48 and $72 \mathrm{~h}$ after A375 cells were transfected with siPLCB2 and PLCB2 expression plasmids. GAPDH was used as a load control. Data are presented as the mean \pm standard deviation. ${ }^{* *} \mathrm{P}<0.01$ vs. the Ctrl group and Ctrl_NC group; ${ }^{\# \#} \mathrm{P}<0.01$ vs. the siPLCB2 group. PLCB2, phospholipase C $\beta 2$; NS, not significant.

A
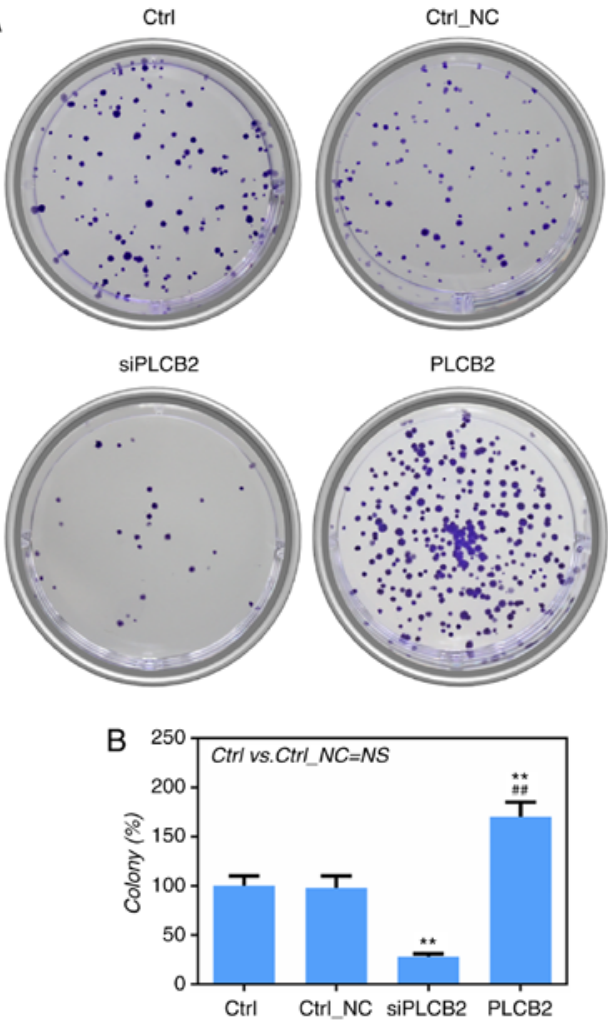

C
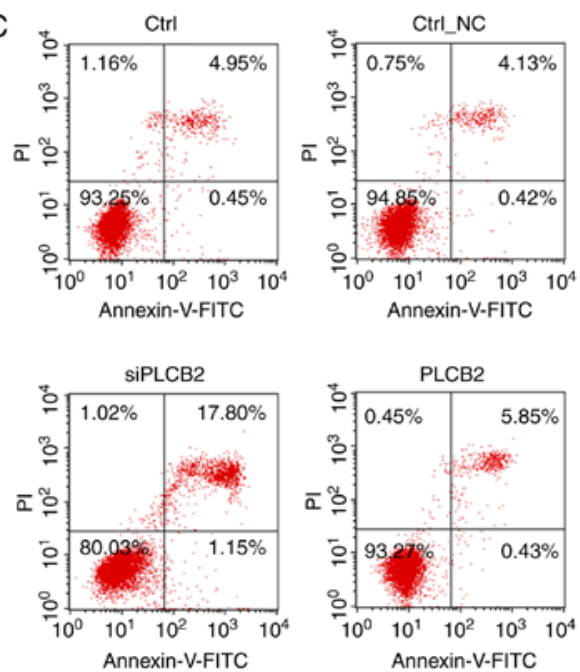

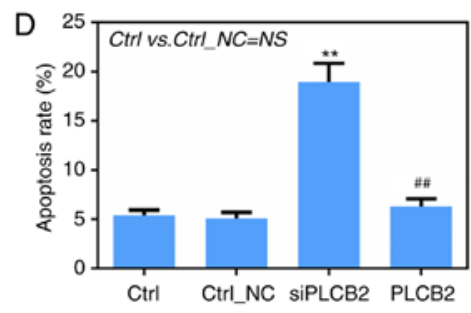

Figure 3. PLCB2 affects cell viability and apoptosis. (A) The cell viability ability was determined by colony formation assay. (B) Histogram of the colony formation efficiency. (C) The apoptosis rate was detected by flow cytometric analysis. (D) Histogram of the apoptosis rate. GAPDH was used as a load control. Data are presented as the mean \pm standard deviation. ${ }^{* * *} \mathrm{P}<0.01$ vs. the $\mathrm{Ctrl}$ group and $\mathrm{Ctrl} \_\mathrm{NC}$ group; ${ }^{\# \#} \mathrm{P}<0.01$ vs. the siPLCB2 group. PLCB2, phospholipase $\mathrm{C} \beta 2$; siPLCB2, PLCB2 interference plasmid; PLCB2, PLCB2 overexpression plasmid. NS, not significant.

A significant decrease in the cell viability was noted in the A375 cells treated with the PLCB2-siRNAs (siPLCB2) when compared to the controls at 24,48 and $72 \mathrm{~h}$ after transfection (100, 79.3 and $58.2 \%$, respectively) (Fig. 2B), while cell viability in the A375 cells treated with the PLCB2 plasmid (PLCB2) was significantly increased $(100,178.5$ and $207.4 \%$, respectively) (Fig. 2B). The role of $P L C B 2$ in the regulation of the colony-formation ability of A375 cells was investigated.
The results showed that low expression of $P L C B 2$ significantly suppressed cell growth and that high expression of $P L C B 2$ significantly promoted cell growth (Fig. 3A and B). Flow cytometric analysis indicated that siPLCB2 significantly enhanced the apoptotic rate of the A375 cells (Fig. 3C and D).

PLCB2 regulates cell viability and the expression levels of apoptosis-related proteins. To detect the mRNA and protein 

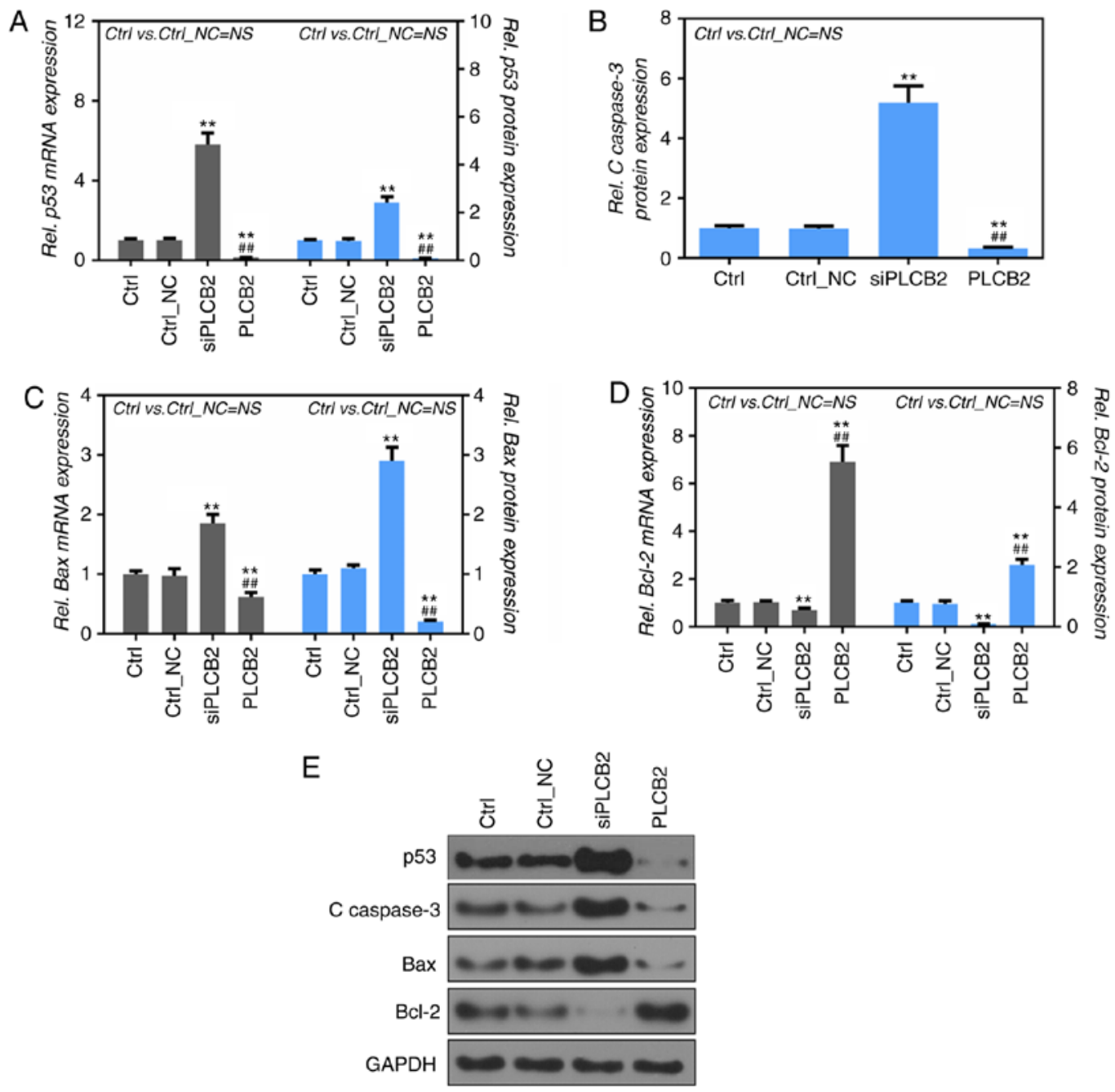

Figure 4. PLCB2 regulates the expression levels of cell viability and apoptosis-related genes. Histogram of the mRNA and protein expression levels of (A) p53, (B) cleaved caspase-3, (C) Bax and (D) Bcl-2. (E) The protein expression levels of p53, cleaved caspase-3, Bax and Bcl-2 were detected by western blot analysis. GAPDH was used as a load control. Data are presented as the mean \pm standard deviation. ${ }^{* *} \mathrm{P}<0.01$ vs. the Ctrl group and Ctrl_NC group; ${ }^{\# \#} \mathrm{P}<0.01$ vs. the siPLCB2 group. PLCB2, phospholipase C $\beta 2$; siPLCB2, PLCB2 interference plasmid; PLCB2, PLCB2 overexpression plasmid. NS, not significant.

expression levels of cleaved caspase-3, Bax and Bcl-2, RT-PCR and western blot analysis were performed. p53, cleaved caspase-3 and Bax mRNA and protein expression levels were significantly increased in the siPLCB2 group, compared to the Ctrl and Ctrl_NC groups ( $\mathrm{P}<0.01$, Fig. $4 \mathrm{~A}-\mathrm{C}$ and $\mathrm{E})$. It was also found that the $\mathrm{Bcl}-2$ protein expression level was significantly upregulated in the PLCB2 group compared with the Ctrl and Ctrl_NC groups $(\mathrm{P}<0.01$, Fig. 4D), while the $p 53$, cleaved caspase-3, Bax and Bcl-2 protein levels showed a reverse trend in the PLCB2 group (Fig. 4A-D).

PLCB2 affects the activation of the Ras/Raf/MAPK signaling pathway. Fig. 5A, B and D shows that the protein levels of Ras and Raf were significantly lower in the siPLCB2 group than that noted in the Ctrl and Ctrl_NC groups and that the protein levels of Ras and Raf were significantly higher in the PLCB2 group than in the Ctrl and Ctrl_NC groups, as determined by western blot analysis $(\mathrm{P}<0.01)$. Furthermore, PLCB2 silencing significantly suppressed the ratio of phosphorylated (p)-ERK1/2/total ERK1/2, and PLCB2 overexpression significantly upregulated the phosphorylation levels of ERK1/2 (P<0.01, Fig. 5C and D).

\section{Discussion}

Due to a deeper understanding and an increasing number of studies on gene variations in cancer, gene therapy is gradually being used to treat cancer. In recent years, some targeted drug therapies have significantly affected the treatment of melanoma (27). However, some patients with melanoma are insensitive to targeted therapy drugs, further causing preexisting resistance (28). Accordingly, it is a key task for melanoma researchers to search for novel prognostic markers, to improve early diagnostic rates, and to identify new targets of molecular-targeted therapy for melanoma. ATP-binding cassette subfamily B member 5 (ABCB5) plays a key role in cellular biological functions in melanoma (29), breast cancer (30), liver cancer (31), oral cancer (32) and colorectal cancer (33). A previous report showed that ABCB5 could regulate melanoma growth and metastasis. These results suggest that ABCB5-related genes might also play a role in cell viability, apoptosis and metastasis in melanoma (29). Bioinformatics analysis could suggest the possible functions of many different novel genes. The GEO database (GSE38290) (18) was analyzed 

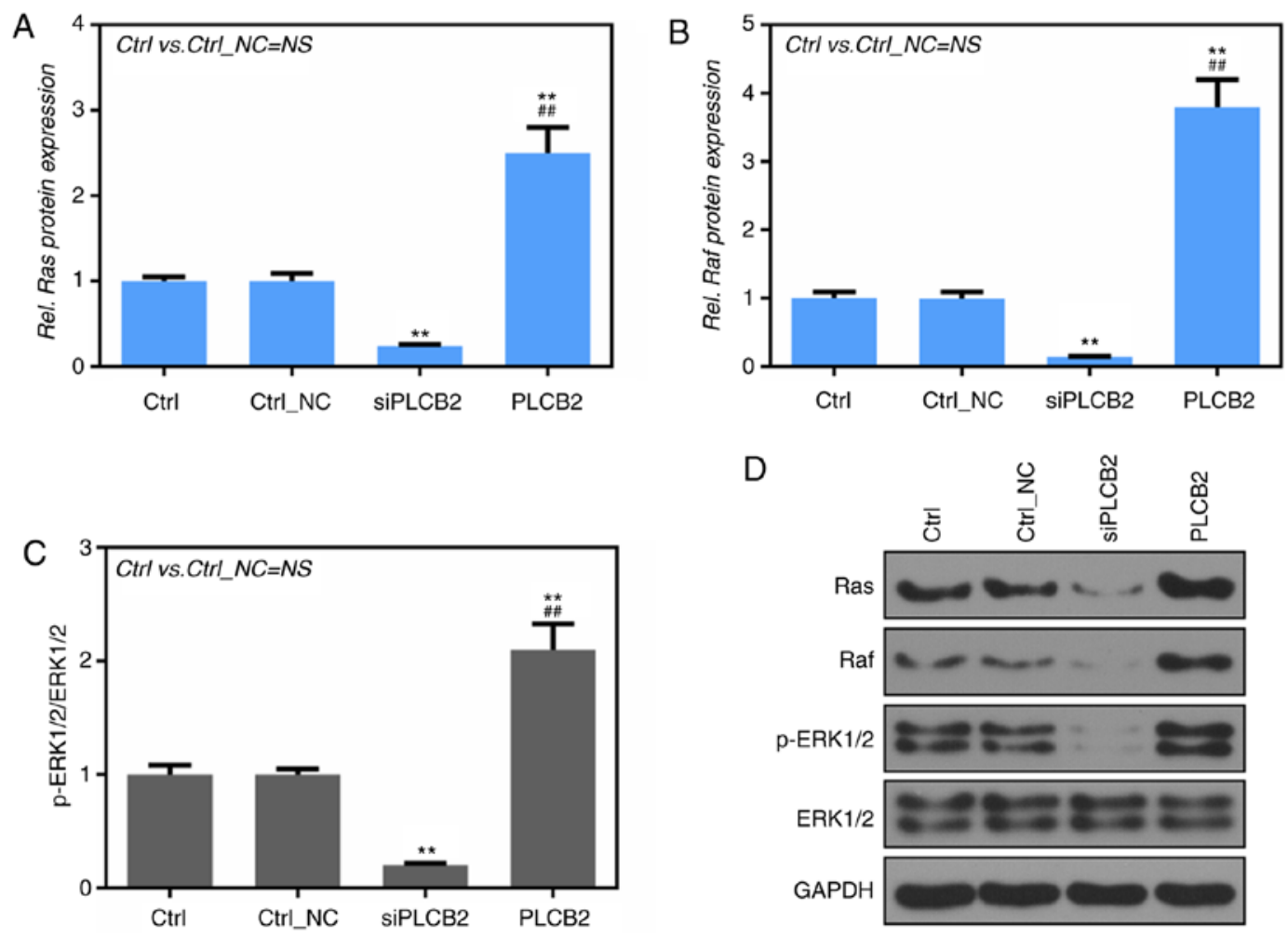

Figure 5. PLCB2 affects activation of the Ras/Raf/MAPK signaling pathway. Histogram of the protein expression level of (A) Ras, (B) Raf and (C) the phosphorylation level of ERK1/2 protein. (D) The protein expression levels of Ras, Raf, ERK1/2 and phosphorylated (p)-ERK1/2 were detected by western blot analysis. GAPDH was used as a load control. Data are presented as the mean \pm standard deviation. ${ }^{* *} \mathrm{P}<0.01$ vs. the Ctrl group and Ctrl_NC group; ${ }^{\# \#} \mathrm{P}<0.01$ vs. the siPLCB2 group. PLCB2, phospholipase C $\beta 2$; siPLCB2, PLCB2 interference plasmid; PLCB2, PLCB2 overexpression plasmid. NS, not significant.

by bioinformatics. The authors identified 345 differentially expressed genes (DEGs), 289 of which were significantly upregulated and 56 of which were significantly downregulated ( $\mathrm{pP}<0.05$, fold-change $>2$ ) in $A B C B 5$ highly expressing human melanoma cell lines (A375 and G3361) (Fig. 1A and B). After gene integration, 13 differentially expressed genes (DEGs) were determined to be closely associated with cancer and to regulate the MAPK cascade (Fig. 1C-E). In addition, further study revealed that $P L C B 2$ was the crucial gene in the PPI network (Fig. 1F and G). In other words, the expression level of $P L C B 2$ was significantly increased after melanoma cells were transfected with the $A B C B 5$ gene. Thus, the authors conclude that $P L C B 2$ may play an important role in cell viability and apoptosis. PLCB2 was significantly upregulated in human melanoma cell lines (Fig. 1H). Given the above results, the expression of PLCB2 was higher in 451-Lu and SK-Mel-28 cells than that noted in the other cell lines and the expression of $P L C B 2$ was lower in Mel Ju and Mel 928 cells than that noted in the other melanoma cell lines, and the expression of $P L C B 2$ in A375 cells was between. Therefore, A375 cells were used in the following experiments.

In the present study, the cell viability ability was determined by CCK- 8 and colony-forming assays and then the cell apoptosis rate was detected by flow cytometry. It was found that $P L C B 2$ interference significantly suppressed cell viability and promoted cell apoptosis (Figs. 2B and 3). As previously shown, PLCB2 affects the associated biological functions in melanoma (29). Thus, the following experiments were performed to preliminarily explore the relevant molecular mechanisms in human melanoma cells overexpressing or not overexpressing $P L C B 2$. According to the above results, $P L C B 2$ has a close relation with the $M A P K$ cascade in melanoma, and the Ras/Raf/MAPK signaling pathway, which belongs to the MAPK signaling transduction pathway, has a close association with skin malignant tumors $(15,17)$. Therefore, it is important to investigate variations in proteins in the Ras/Raf/MAPK signaling pathway. Ras and $p 53$ are crucial genes involved in cell proliferation, cell apoptosis, cell movement, the inflammatory response and angiogenesis (34). There is cooperation between the Ras and $p 53$ genes. Accordingly, the expression level of $p 53$ was detected by RT-PCR and western blot analysis. $P L C B 2$ interference significantly increased the mRNA and protein levels of $p 53$, and the overexpression of $P L C B 2$ significantly decreased the mRNA and protein levels of $p 53$ (Fig. 4A). Ras expression changes in A375 cells overexpressing or not overexpressing PLCB2 were the same as those changes noted for $p 53$ expression (Fig. 5A). This result is consistent with those that have been well documented. The MAPK signaling pathway regulates $B a x$ and $B c l-2$ gene expression and actives caspase-3 expression (35). In the present study, $P L C B 2$ interference significantly enhanced cleaved caspase-3 and $B a x$ gene expression and suppressed $B c l-2$ gene expression (Fig. 4). Guanosine triphosphate (GTP)-Ras was found to activate $R a f$ gene expression to activate $M E K / E R K$ signaling (36). According to a previous report, Ras can activate the phosphorylation level of ERK1/2 in the MAPK signaling pathway to regulate cell apoptosis $(36,37)$. The results described here revealed that PLCB2 interference suppressed the activation 
of the Ras/Raf/MAPK signaling pathway, as demonstrated by western blot analysis (Fig. 5).

In summary, $P L C B 2$ may be a potential target gene in the treatment of melanoma and could affect the activation of the Ras/Raf/MAPK signaling pathway to regulate the expression levels of $B c l-2$, Bax, caspase-3 and p53, thereby affecting cell viability and apoptosis.

\section{Acknowledgements}

Not applicable.

\section{Funding}

The present study was funded by The Medical and Health Science and Technology Project of Zhejiang Province to HZ (grant no. 2017KY070).

\section{Availability of data and materials}

GSE38290 dataset (18) was used to perform our study. The datasets generated and/or analyzed during the current study are available from the Gene Expression Omnibus, https:// www.ncbi.nlm.nih.gov/geo/.

\section{Authors' contributions}

HZ wrote the main manuscript and analyzed the data. TX and YS performed all the experiments and collected the data. HZ and YQ designed the study. All authors read and approved the final manuscript and agree to be accountable for all aspects of the research in ensuring that the accuracy or integrity of any part of the work are appropriately investigated and resolved.

\section{Ethics approval and consent to participate}

Not applicable.

\section{Patient consent for publication}

Not applicable.

\section{Competing interests}

The authors declare that they have no competing interests.

\section{References}

1. Little EG and Eide MJ: Update on the current state of melanoma incidence. Dermatol Clin 30: 355-361, 2012.

2. Eggen CAM, Durgaram VVL, van Doorn R, Mooi WJ, Pardo LM, Pasmans SGMA and Hollestein LM: Incidence and relative survival of melanoma in children and adolescents in the Netherlands, 1989-2013. J Eur Acad Dermatol Venereol 32: 956-961, 2018.

3. Aung PP, Nagarajan P and Prieto VG: Regression in primary cutaneous melanoma: Etiopathogenesis and clinical significance. Lab Invest: Feb 27, 2017 (Epub ahead of print). doi: 10.1038/labinvest.201.

4. Lorigan JG, Wallace S and Mavligit GM: The prevalence and location of metastases from ocular melanoma: Imaging study in 110 patients. AJR Am J Roentgenol 157: 1279-1281, 1991.
5. Yang M, Jiang $\mathrm{P}$, An Z, Baranov E, Li L, Hasegawa S Al-Tuwaijri M, Chishima T, Shimada H, Moossa AR and Hoffman RM: Genetically fluorescent melanoma bone and organ metastasis models. Clin Cancer Res 5: 3549-3559, 1999.

6. Seifert H, Hirata E, Gore M, Khabra K, Messiou C, Larkin J and Sahai E: Extrinsic factors can mediate resistance to BRAF inhibition in central nervous system melanoma metastases. Pigment Cell Melanoma Res 29: 92-100, 2016.

7. Bedikian AY, Papadopoulos NE, Kim KB, Vardeleon A, Smith T, Lu B and Deitcher SR: A pilot study with vincristine sulfate liposome infusion in patients with metastatic melanoma. Melanoma Res 18: 400-404, 2008.

8. Elder DE: Pathology of melanoma. Surg Oncol Clin N Am 24: 229-237, 2015.

9. Amann VC, Ramelyte E, Thurneysen S, Pitocco R, Bentele-Jaberg N, Goldinger SM, Dummer R and Mangana J: Developments in targeted therapy in melanoma. Eur J Surg Oncol 43: 581-593, 2017.

10. Mao G, Jin J, Kunapuli SP and Rao AK: Nuclear factor- $\kappa B$ regulates expression of platelet phospholipase C-beta2 (PLCB2). Thromb Haemost 116: 931-940, 2016.

11. Suire S, Lecureuil C, Anderson KE, Damoulakis G, Niewczas I, Davidson K, Guillou H, Pan D, Jonathan Clark, Phillip T Hawkins and Stephens L: GPCR activation of Ras and PI3Kc in neutrophils depends on PLCb2/b3 and the RasGEF RasGRP4. EMBO J 31: 3118-3129, 2012.

12. Chen G, Wang Y, Wang L and Xu W: Identifying prognostic biomarkers based on aberrant DNA methylation in kidney renal clear cell carcinoma. Oncotarget 8: 5268-5280, 2017.

13. Zekri AR, Hassan ZK, Bahnassy AA, Khaled HM, El-Rouby MN, Haggag RM and Abu-Taleb FM: Differentially expressed genes in metastatic advanced Egyptian bladder cancer. Asian Pac J Cancer Prev 16: 3543-3549, 2015.

14. Collisson EA, De A, Suzuki H, Gambhir SS and Kolodney MS: Treatment of metastatic melanoma with an orally available inhibitor of the Ras-Raf-MAPK cascade. Cancer Res 63: 5669-5673, 2003.

15. Poenitzsch Strong AM, Setaluri V and Spiegelman VS: MicroRNA-340 as a modulator of RAS-RAF-MAPK signaling in melanoma. Arch Biochem Biophys 563: 118-124, 2014.

16. Barault L, Veyrie N, Jooste V, Lecorre D, Chapusot C, Ferraz JM, Lièvre A, Cortet M, Bouvier AM, Rat $\mathrm{P}$, et al: Mutations in the RAS-MAPK, PI(3)K (phosphatidylinositol-3-OH kinase) signaling network correlate with poor survival in a population-based series of colon cancers. Int J Cancer 122: 2255-2259, 2008.

17. Li XJ, Li B, Huang JS, Shi JM, Wang P, Fan W and Zhou YL: Effects of acrylonitrile on lymphocyte lipid rafts and RAS/RAF/MAPK/ERK signaling pathways. Genet Mol Res 13: 7747-7756, 2014

18. Wilson BJ, Saab KR, Ma J, Schatton T, Pütz P, Zhan Q, Murphy GF, Gasser M, Waaga-Gasser AM, Frank NY and Frank MH: ABCB5 maintains melanoma-initiating cells through a proinflammatory cytokine signaling circuit. Cancer Res 74: 4196-207, 2014.

19. Subramanian A, Tamayo P, Mootha VK, Mukherjee S, Ebert BL, Gillette MA, Paulovich A, Pomeroy SL, Golub TR, Lander ES and Mesirov JP: Gene set enrichment analysis: A knowledge-based approach for interpreting genome-wide expression profiles. Proc Natl Acad Sci USA 102: 15545-15550, 2005.

20. Gao X, Wang X and Zhang S: Bioinformatics identification of crucial genes and pathways associated with hepatocellular carcinoma. Biosci Rep 38, 2018:

21. Qin J, Fang N, Lou J, Zhang W, Xu S, Liu H, Fang Q, Wang Z, Liu J, Men X, et al: TRB3 is involved in free fatty acid-induced INS-1-derived cell apoptosis via the protein kinase C delta pathway. PLoS One 9: e96089, 2014.

22. Mohan N, Ai W, Chakrabarti M, Banik NL and Ray SK: KLF4 overexpression and apigenin treatment down regulated anti-apoptotic $\mathrm{Bcl}-2$ proteins and matrix metalloproteinases to control growth of human malignant neuroblastoma SK-N-DZ and IMR-32 cells. Mol Oncol 7: 464-474, 2013.

23. Flores-Fernandez R, Blanco-Favela F, Fuentes-Panana EM, Chavez-Sanchez L, Gorocica-Rosete P and Chávez-Rueda AK: Prolactin rescues immature B-cells from apoptosis induced by B-cell receptor cross-linking. J Immunol Res 2016: 3219017, 2016.

24. Zhang XD, Wu Q and Yang SH: Ferulic acid promoting apoptosis in human osteosarcoma cell lines. Pak J Med Sci 33: 127-131, 2017. 
25. Livak KJ and Schmittgen TD: Analysis of relative gene expression data using real-time quantitative PCR and the 2(-Delta Delta C(T)) method. Methods 25: 402-408, 2001.

26. Zhao L, Gu H, Chang J, Wu J, Wang D, Chen S, Yang X and Qian B: MicroRNA-383 regulates the apoptosis of tumor cells through targeting Gadd45g. PLoS One 9: e110472, 2014.

27. Queirolo $P$ and Spagnolo F: BRAF plus MEK-targeted drugs: A new standard of treatment for BRAF-mutant advanced melanoma. Cancer Metastasis Rev 36: 35-42, 2017.

28. Ahn A, Chatterjee A and Eccles MR: The slow cycling phenotype: A growing problem for treatment resistance in melanoma. Mol Cancer Ther 16: 1002-1009, 2017.

29. Wang S, Tang L, Lin J, Shen Z, Yao Y, Wang W, Tao S, Gu C, Ma J, Xie Y and Liu Y: ABCB5 promotes melanoma metastasis through enhancing NF- $\kappa \mathrm{B}$ p65 protein stability. Biochem Biophys Res Commun 492: 18-26, 2017.

30. Yao J, Yao X, Tian T, Fu X, Wang W, Li S, Shi T, Suo A, Ruan Z, Guo $\mathrm{H}$, et al: ABCB5-ZEB1 axis promotes invasion and metastasis in breast cancer cells. Oncol Res 25: 305-316, 2017.

31. Chong CC, Cheung ST, Cheung YS, Chan AW, Chan SL, Yu SC and Lai PB: Novel biomarkers GEP/ABCB5 regulate response to adjuvant transarterial chemoembolization after curative hepatectomy for hepatocellular carcinoma. Hepatobiliary Pancreat Dis Int 17: 524-530, 2018

32. Grimm M, Krimmel M, Polligkeit J, Alexander D, Munz A, Kluba S, Keutel C, Hoffmann J, Reinert S and Hoefert S: ABCB5 expression and cancer stem cell hypothesis in oral squamous cell carcinoma. Eur J Cancer 48: 3186-3197, 2012.
33. Guo Q, Grimmig T, Gonzalez G, Giobbie-Hurder A, Berg G, Carr N, Wilson BJ, Banerjee P, Ma J, Gold JS, et al: ATP-binding cassette member B5 (ABCB5) promotes tumor cell invasiveness in human colorectal cancer. J Biol Chem 293: 11166-11178, 2018.

34. Zhang X, Cheng Q, Yin H and Yang G: Regulation of autophagy and EMT by the interplay between p53 and RAS during cancer progression (Review). Int J Oncol 51: 18-24, 2017.

35. Yousefi H, Karimi P, Alihemmati A, Alipour MR, Habibi P and Ahmadiasl N: Therapeutic potential of genistein in ovariectomy-induced pancreatic injury in diabetic rats: The regulation of MAPK pathway and apoptosis. Iran J Basic Med Sci 20: 1009-1015, 2017

36. Ritt DA, Abreu-Blanco MT, Bindu L, Durrant DE, Zhou M, Specht SI, Stephen AG, Holderfield $M$ and Morrison DK: Inhibition of Ras/Raf/MEK/ERK pathway signaling by a stress-induced phospho-regulatory circuit. Mol Cell 64: 875-887, 2016

37. Han YF, Ji LH, Feng TT, Liu F, Cui S and Su J: Effect of ERK1/2 signaling pathway inhibitor PD98059 on the expression of ras, BRaf, MEK, ERK1/2 in marrow nucleated red blood cells of CMS Patients. Zhongguo Shi Yan Xue Ye Xue Za Zhi 25: 1571-1575 (In Chinese). 